EPJ manuscript No.

(will be inserted by the editor)

\title{
A limited resource model of fault-tolerant capability against cascading failure of complex network
}

\author{
Ping $\mathrm{Li}^{1}$, Bing-Hong Wang ${ }^{2,3}$, Han $\mathrm{Sun}^{1}$, Pan $\mathrm{Gao}^{1}$, Tao Zhou ${ }^{2,4}$ \\ 1 Department of Basic Sciences, Nanjing Institute of Technology, Nanjing 211167, China \\ 2 Department of Modern Physics and Nonlinear Science Center, University of Science and Technology of China, Hefei 230026, \\ China \\ 3 Shanghai Academy of System Science, Shanghai, 200093 China \\ 4 Department of Physics, University of Fribourg, CH-1700 Fribourg, Switzerland
}

Received: date / Revised version: date

\begin{abstract}
We propose a novel capacity model for complex networks against cascading failure. In this model, vertices with both higher loads and larger degrees should be paid more extra capacities, i.e. the allocation of extra capacity on vertex $i$ will be proportional to $k_{i}^{\gamma}$, where $k_{i}$ is the degree of vertex $i$ and $\gamma>0$ is a free parameter. We have applied this model on Barabási-Albert network as well as two real transportation networks, and found that under the same amount of available resource, this model can achieve better network robustness than previous models.
\end{abstract}

PACS. 89.75.Hc Networks and genealogical trees - 05.10.-a Computational methods in statistical physics and nonlinear dynamics

\section{Introduction}

Many systems in nature and society can be described by networks, including biological and social systems, the Internet, the world-wide web, friendship networks, computer networks, metabolic networks, power grids, scientific citations, neural networks, and so on. Therefore, complex networks have recently attracted considerable attention in physics and other fields. Interestingly, many real-world networks share a certain number of common topological properties, such as small-world and scale-free properties [1,2,3,4. The network robustness is one of the central topics in studying of complex networks. Robustness refers to the malfunction avoiding ability of a network when a fraction of its constituents are damaged. Previous works demonstrated that the heterogeneity of a network induces a high resilience to random failure while a high sensitivity to intentional attacks [5,6,7]. A common failure of many networks is cascading failure triggered by the removal of vertices or overload breakdown of vertices. The robustness of complex networks in response to cascading failure of intentional attacks has become a topic of recent interest. Prior studies have shown that the fault-tolerant capability of network have a great impact on their robustness and function $5,5,6,7,8,9,10,11,12,13,14,15,16,17,18,19,20,21$, 22, 23, 24, 25, 26, 27, 28, 29, 30, 31, 32, 33, 34, 35, 36.

Any failures of vertices in general will change the distribution of loads. Here the load of a vertex (also called betweenness centrality 37,38 ) is defined as the total number of shortest paths passing through this vertex. If the load at a particular vertex increases and becomes larger than its capacity, the corresponding vertex fails. The overload failure leads to redistribution about the loads and, as a result, subsequently cascading failures may occur. Because of the global redistribution of load, new overload failures may be driven by events happening far away. This cascading process may stop after a few steps, while it could also spread over the entire network.

The damage caused by cascading failures can be quantified by the relative size of the largest connected component $G$, defined as following

$$
G=N^{\prime} / N,
$$

where $N$ and $N^{\prime}$ are the numbers of vertices in the largest component before and after the cascade, respectively. The integrity of a network is maintained if $G \approx 1$, while breakdown occurs if $G \approx 0$. The relative size $G$ also represents the robustness of complex networks against cascading failures. The cascading failure can be prevented by assigning extra capacities to vertices. Since the extending of network capacity will bring economic and technique pressure, it is important to explore how to rationally allocate the limited capacity onto vertices, and efficiently improve the robustness of network. The capacity of a vertex is the maximum load that the vertex can handle. Assuming the capacity $C_{i}$ of vertex $i$ be proportional to its initial load $L_{i}$ :

$$
C_{i}=\lambda_{i} \cdot L_{i},
$$




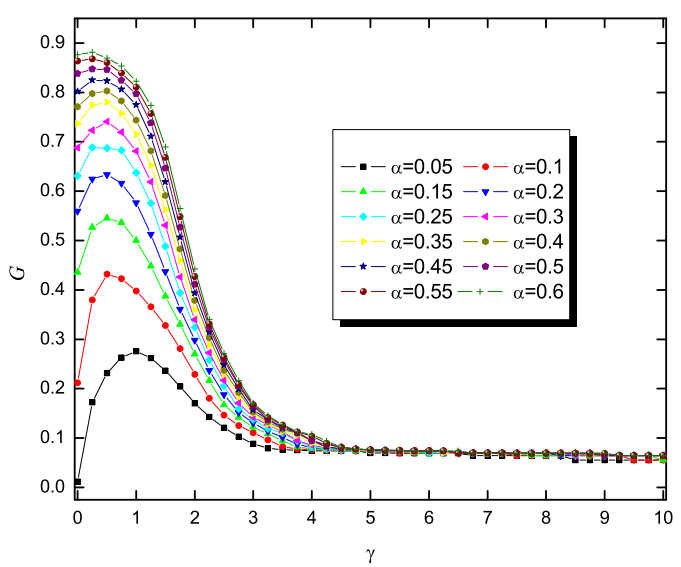

Fig. 1. The robustness $G$ vs. $\gamma$ under attacking of the highestload vertex on BA network of size $N=5000$ and average degree $\langle k\rangle=4$ for different $\alpha$.

where $\lambda_{i}>1$ is the tolerance parameter. Excess term $\left(\lambda_{i}-\right.$ $1)$ is the extra capacity of vertex $i$, which simultaneously reflects $i$ 's ability of tolerating the additional burden and the extra cost to protect $i$.

\section{Model}

Among the previous works, two models should be paid special attention: Motter-Lai (ML) model [10] and WangKim (WK) model 33. ML model assumes the capacity $C_{i}$ of vertex $i$ be proportional to the initial load $L_{i}$ as

$$
C_{i}=(1+\alpha) \cdot L_{i}, \quad i=1,2, \ldots, N
$$

where $\alpha \geq 0$ is the control parameter representing the extra capacity. In WK model, the capacity $C_{i}$ is

$$
C_{i}=\left(1+\alpha \cdot \Theta\left(\frac{L_{i}}{L_{\max }}-\beta\right)\right) \cdot L_{i}, i=1,2, \ldots, N,
$$

where $\Theta(x)=0(1)$ for $x<0(>0)$ is a two-valued function, namely the Heaviside step function, $L_{\max }=\max _{i}\left(L_{i}\right)$, $\alpha \in[0, \infty)$ and $\beta \in[0,1]$ are two control parameters. Here, each vertex can be in one of two states: assigned some extra capacity, or not. When $\beta=0$, WK model degenerates to ML model. Since fault-tolerant capacity is costly [5, 10, 17, 18, 33], a fundamental concern is how to efficiently allocate limited resources of capacity to makes network more robust. ML model raises a linear correlation between extra capacity and initial load, while WK model prefers to protect the highest-load vertices. We agree with WK model that under the same cost, a certain more heterogeneous distribution of extra capacities can further enhance the robustness, however, the two-step function may be oversimplified. According to the previous works [10 33, the total cost $e$ to protect a network can be defined as:

$$
e=\frac{1}{N} \sum_{i=0}^{N}\left(\lambda_{i}-1\right)
$$

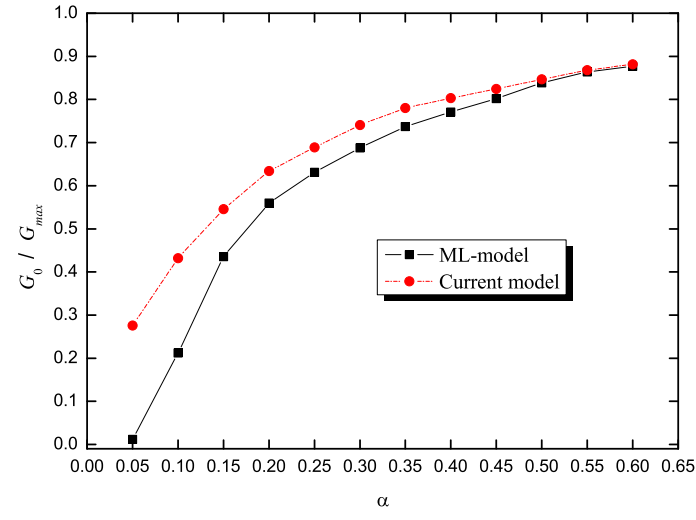

Fig. 2. $G_{0}$ and $G_{\max }$ vs. $\alpha$.

In ML model, the cost is

$$
e_{M L}=\frac{1}{N} \sum_{i=0}^{N}\left(\lambda_{i}-1\right)=\frac{1}{N} \sum_{i=0}^{N}(\alpha)=\alpha .
$$

In the WK model, the cost is

$e_{W K}=\frac{1}{N} \sum_{i=0}^{N}\left(\lambda_{i}-1\right)=\frac{1}{N} \sum_{i=0}^{N} \alpha \cdot \Theta\left(\frac{L_{i}}{L_{\max }}-\beta\right)=\alpha \cdot \frac{N^{\prime \prime}}{N}$,

where $N^{\prime \prime}$ is the number of vertices with initial load larger than $\beta L_{\max }$.

In this paper, we propose a novel allocation mechanism of limited resources of capacity against cascading failures, as:

$$
C_{i}=\left(1+\alpha k_{i}^{\gamma} /\left\langle k^{\gamma}\right\rangle\right) \cdot L_{i}
$$

where $\alpha \geq 0$ and $\gamma \geq 0$ are two free parameters, and

$$
\left\langle k^{\gamma}\right\rangle=\frac{1}{N} \sum_{i=1}^{N} k_{i}^{\gamma}
$$

The parameter $\alpha$ corresponds to the extra capacity, and $\gamma$ is the parameter controlling the heterogeneity of resource allocation. the cost of this model is

$$
e=\frac{1}{N} \sum_{i}^{N}\left(\lambda_{i}-1\right)=\frac{1}{N} \sum_{i}^{N} \alpha k_{i}^{\gamma} /\left\langle k^{\gamma}\right\rangle=\alpha .
$$

Different from ML model, the fault-tolerant capacity and topological structure are interrelated in the current model. Since the components of a network may have different fault-tolerant capacities, this work raises a more general allocation strategy for extra capacity. The assigning extra capacity depends not only on load $L_{i}$ of vertex $i$ but also on the number of its links. When $\gamma>0$, vertices with larger degrees could be allocated more extra capacities. The case $\gamma=0$ degenerates to ML model, and the case $\gamma \rightarrow \infty$ represents the extremely heterogeneous allocation where only the most connected vertex is protected. 


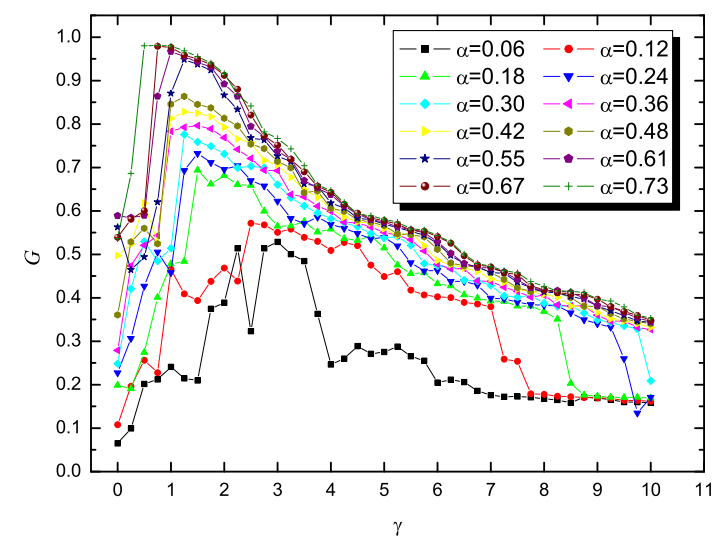

Fig. 3. The robustness $G$ vs. $\gamma$ under attacking of the highestload vertex for UPTN on Beijing with size $N=4127$ for different $\alpha$.

\section{Simulation}

Fig. 1 reports the robustness $G$ as a function of $\gamma$ at several typical values of $\alpha$ for BA network 39, 40 of size $N=5000$ and average degree $\langle k\rangle=4$. We find that the robustness has a maximum, $G_{\max }$, with $\gamma \in(0,1)$ for different $\alpha$, indicating that the allocation of the limited resource of fault-tolerant capability should be neither uniform nor extremely uneven, but a little bit more heterogenous (for $\gamma>0$ ) than the case of ML model. When $\gamma \geq 5$, all $G(\gamma)$ at different $\alpha$ converge to a single curve, indicating that the extremely uneven distribution of resource, with the largest-degree node possessing the majority of extra capacity, has even worse performance then the uniform allocation (except for the cases with $\alpha$ close to zero). Define $G_{0}=G(\gamma=0)$, and $G_{\max }=\max _{\gamma} G(\gamma)$ for given $\alpha$. The former corresponds to the ML model, while the latter to the best robustness predicted by the current model. In Fig. 2, we plot $G_{0}$ and $G_{\max }$ vs. $\alpha$ for the same data in Fig. 1. Clearly, in a broader region of $\alpha$, $G_{\max }$ is always greater than $G_{0}$, demonstrating that we can get higher robustness of network than ML model with the same amount of resource.

The serious global failure, as a catastrophe resulting from cascading propagation 13, has often been observed in some kinds of traffic networks suffering overload failure, where the word "traffic" can stand for information packets of the Internet [41,42, the electronic flow in the power grids 43], as well as passengers in public transportation systems [4, 45. Next, we compare the performance of the current model with ML model on some real networks. Especially, the drawing near Olympic 2008 in Beijing and World Expo 2010 in Shanghai will bring extremely heavy traffic pressure on Beijing and Shanghai urban traffic systems, thus an urgent problem is how to allocate the limited additional traffic capacities to avoid possible cascading traffic congestions. The topological characters of urban public traffic networks (UPTNs) are reported previously

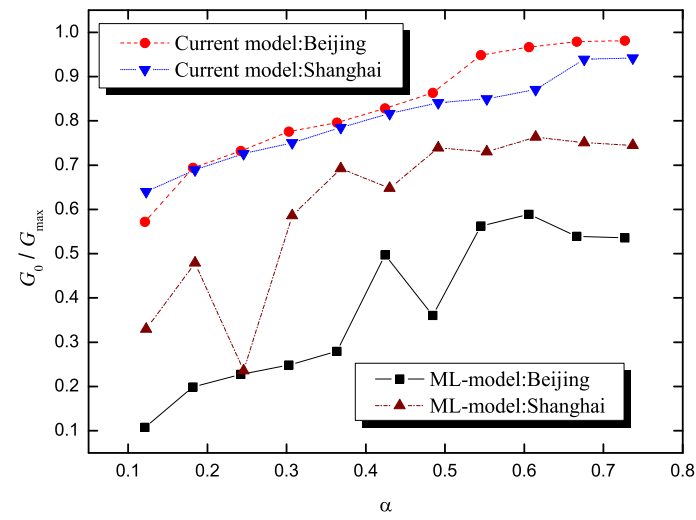

Fig. 4. $G_{0}$ and $G_{\max }$ vs. $\alpha$. for UPTNs in Beijing $(N=4127)$ and Shanghai $(\mathrm{N}=2035)$.

[46], and here we investigate the same data to see if our model can achieve better performance than ML model.

In Fig.3, we show the relation between the robustness $G$ of Beijing's UPTN and the parameter $\gamma$ of allocation of capacity resources at various values of $\alpha$. Qualitatively speaking, the pattern of $G(\gamma)$ is similar to that of BA network. Fig. 4 compared the performance of the current model and ML model for UPTNs in Beijing and Shanghai respectively. Clearly, the current model can always perform better than ML model under the same amount of extra capacity. Moreover, large fluctuation is observed for ML model, thus one could not guarantee the enhancement of robustness giving more resource. In contrast, $G_{\max }$ increases with $\alpha$ monotonously in the current model. Two $G(\alpha)$ curves in Beijing and Shanghai are remarkably different for ML model, while almost have almost the same trend for the current model. Perhaps, this indicates that current model has some universality to improve robustness for different real-world networks, or maybe it is because the robustness of this strategy is near the optimal one.

\section{Summary}

In summary, the robustness of complex networks is analyzed within the framework of allocating extra fault-tolerant capacity against cascading failure. We proposed a novel capacity model, which achieves higher robustness for the same amount of resource as ML model [10. In previous works, the extra capacity of a vertex is linearly correlated with its load, or simply divided into two discrete values by a step function. In this paper, we argue that those prior strategies may be oversimplified, and a more complicated and more heterogenous (than ML model) allocation strategy could further enhance the robustness. The simulation results of BA networks and two typical real transportation networks strongly support the efficiency of our strategy. It is worthwhile to emphasize two features of the current model: Firstly, the maximum value of robustness $G_{\max }$ 
is a monotonously increasing function on parameter $\alpha$, in contrast with ML model. Secondly, the same trend and pattern of $G_{\max }(\alpha)$ for different UPTNs indicates that this model may have some universality for robustness improvement of different real-world networks (Maybe it is because the performance of this strategy is near the optimal one). However, this point is not clear thus far, which needs further investigation. Besides, although the previous works suggested using the mean relative extra capacity $e$ to measure the cost, it is also, to some extent, reasonable to measure the cost by using absolute extra capacity. In that way, given $\alpha$, the cost will be a monotonous function of $\gamma$, and our numerical results give raise to a practically significant conclusion: There exists a specific threshold of $\gamma_{c}$ corresponding to $G_{\max }$, below which one could improve the robustness by spending more, while above which the additional resource will, in contrast to what we expect, makes the network worse robust. Although at a first step far from the final goal, optimal allocation, we believe this model have its theoretical importance and potential application in designing infrastructure networks from the point of economic view. It can also provide guidance in designing more robust artificial networks.

This work is funded by the National Basic Research Program of China (973 Project No. 2006CB705500), the National Natural Science Foundation of China (Grant Nos. 10472116, 10532060, and 10635040), and the Science and Technology Foundation (KXJ06048) as well as the Student Foundation (NB2006183) of Nanjing Institute of Technology.

\section{References}

1. M. E. J. Newman, SIAM Review 45, 167 (2003).

2. S. Boccaletti, V. Latora, Y. Moreno, M. Chavez, and D. -U. Hwang, Phys. Rep. 424, 175 (2006).

3. R. Albert and A. -L. Barabsi, Rev. Mod. Phys. 74, 47 (2002).

4. S. N. Dorogovtsev and J. F. F. Mendes, Adv. Phys. 51, 1079 (2002).

5. R. Albert, H. Jeong and A. -L. Barabsi, Nature 406, 378 (2000).

6. R. Cohen, K. Erez, D. ben-Avraham, and S. Havlin, Phys. Rev. Lett. 85, 4626 (2000).

7. R. Cohen, K. Erez, D. ben-Avraham, and S. Havlin, Phys. Rev. Lett. 86, 3682 (2001).

8. P. Holme, B. J. Kim, C. N. Yoon and S. K. Han, Phys. Rev. E 65, 056109 (2002).

9. P. Holme, and B. J. Kim, Phys. Rev. E 65, 066109 (2002).

10. A. E. Motter, and Y. C. Lai, Phys. Rev. E 66, 065102 (R) (2002).

11. A. E. Motter, and T. Nishikawa, Phys. Rev. E 66, 065103 (R) (2002).

12. P. Crucittia, V. Latorab, M. Marchioric, and A. Rapisardab, Physica A 320, 622 (2003).

13. T. Zhou and B. H. Wang, Chin. Phys. Lett. 22, 1072 (2005).

14. Y. C. Lai, A. E. Motter, and T. Nishikawa, Lect. Notes Phys. 650, 299 (2004).
15. J. G. Liu, Z. T. Wang, and Y. Z. Dang, Mod. Phys. Lett. B 19, 785 (2005).

16. J. G. Liu, Z. T. Wang, and Y. Z. Dang, Mod. Phys. Lett. B 20, 815 (2006).

17. L. Zhao, K. Park, and Y. C. Lai, Phys. Rev. E 70, 035101(R) (2004).

18. P. Crucitti, V. Latora, and M. Marchiori, Phys. Rev. E 69, 045104 (2004).

19. A. E. Motter, Phys. Rev. Lett. 93, 098701 (2004).

20. P. Crucitti, V. Latora, M. Marchiori, and A. Rapisarda, Physica A 340, 388 (2004).

21. P. Holme, Europhys. Lett. 68,908 (2004).

22. L. K. Gallos, R. Cohen, P. Argyrakis, A. Bunde, and S. Havlin, Phys. Rev. Lett. 94, 188701 (2005).

23. V. Latora and M. Marchiori, Phys. Rev. E 71, 015103 (2005).

24. E. J. Lee, K. I. Goh, B. Kahng, and D. Kim, Phys. Rev. E 71, 056108 (2005).

25. Y. C. Lai, A. E. Motter, T. Nishikawa, K. Park, and L.Zhao, Pramana-J. Phys. 64, 483 (2005).

26. L. Zhao, K. Park, Y. C. Lai and N. Ye, Phys. Rev. E 72, 025104(R) (2005).

27. R. Kinney, P. Crucitti, R. Albert, and V. Latora Eur. Phys. J. B 46, 101 (2005).

28. L. Dall'Asta, A. Barrat, M. Barthelemy, and A. Vespignani, J. Stat. Mech. Theory and Experiment, P04006 (2006).

29. J. $\Phi$. H. Bakke, A. Hansen, and J. Kertész, Europhys. Lett. 76, 717 (2006).

30. T. Tanizawa, G. Paul, S. Havlin, and H. E. Stanley, Phys. Rev. E 74, 016125 (2006).

31. G. Paul, S. Sreenivasan, S. Havlin, and H. E. Stanley, Physica A 370, 854 (2006).

32. B. Wang, H. W. Tang, Z. L. Xiu, C. H. Guo, and T. Zhou, Physica A 368, 607 (2006).

33. B. Wang and B. J. Kim, EPL 78, 48001 (2007).

34. S. Sreenivasan, R. Cohen, E. López, Z. Toroczkai, and H. E. Stanley, Phys. Rev. E 75, 036105 (2007).

35. Z. Z. Guo, X. W. Wu, and C. A. Wang Pramana-J. Phys. 68, 689 (2007).

36. D. Centola, V. M. Eguíluz, and M. W. Macy, Physica A 374, 449 (2007).

37. L. C. Freeman, Sociometry, 40, 35 (1977).

38. T. Zhou, J. G. Liu, and B. H. Wang, Chin. Phys. Lett. 23 2327 (2006).

39. A. -L. Barabási, and R. Albert, Science 286, 509 (1999).

40. A. -L. Barabási, R. Albert, and H. Jeong, Physica A 272, 173 (1999).

41. G. Yan, T. Zhou, B. Hu, Z. Q. Fu, and B. H. Wang, Phys. Rev. E 73, 046108 (2006).

42. B. H. Wang, and T. Zhou, J. Korean Phys. Soc. 50, 134 (2007).

43. W. J. Bai, T. Zhou, Z. Q. Fu, Y. H. Chen, X. Wu, and B. H. Wang, Proc. ICCCAS'2006 (IEEE Press 2000, pp. 17441748).

44. P. P. Zhang, K. Chen, Y. He, T. Zhou, B. B. Su, Y. D. Jin, H. Chang, Y. P. Zhou, L. C. Sun, B. H. Wang, and D. R. He, Physica A 360, 599 (2006).

45. H. K. Liu, and T. Zhou, Acta Physica Sinica 56, 106 (2007).

46. P. Li, X. Xiong, Z. L. Qiao, G. Q. Yuan, X. Sun, and B. H. Wang, Chin. Phys. Lett. 23, 3384 (2006). 